Supplementary Information

\title{
Nitrogen-Doped Carbon Nanotubes Supported by Macroporous Carbon as an Efficient Enzymatic Biosensing Platform for Glucose
}

Yonghai Song ${ }^{\dagger}$ Xingping Lu, ${ }^{\dagger} \mathrm{Yi} \mathrm{Li},{ }^{\dagger}$ Qiaohui Guo, ${ }^{\dagger}$ Shuiliang Chen,${ }^{\dagger}$ Lanqun Mao, ${ }^{\star}$ Haoqing

$$
\text { Hou, }{ }^{\dagger} \text { and Li Wang }{ }^{*, \dagger}
$$

${ }^{\dagger}$ Key Laboratory of Functional Small Organic Molecule, Ministry of Education, Key Laboratory of Chemical Biology, Jiangxi Province, College of Chemistry and Chemical Engineering, Jiangxi Normal University, Nanchang 330022, China

${ }^{+}$Beijing National Laboratory for Molecular Sciences, Key Laboratory of Analytical Chemistry for Living Biosystems, Institute of Chemistry, Chinese Academy of Sciences, Beijing 100190, China

* Tel/Fax: +86 791 88120861. E-mail: lwangsy2003@hotmail.com 


\section{Contents}

Schematic illustration of the fabrication of the integrated 3D-KSC/NCNTs electrode.......Figure S1

SEM images of 3D-KSC/NCNTs

Figure S2

SEM images of 3D-KSC/Ni NPs

Figure S3

SEM images of 3D-KSC/NCNTs obtained at a growth time of $20 \mathrm{~min}$

Figure S4

CVs and LSV of different electrodes....

Figure S5

Stability test of 3D-KSC/NCNTs nanocomposites in ORR

Figure S6

EIS for 3D-KSC/NCNTs/GOD and 3D-KSC/GOD

Figure S7

Stability and reproducibility of 3D-KSC/NCNTs/GOD in determination of glucose

Figure S8

Selectivity test of 3D-KSC/NCNTs/GOD biosensor.

.Figure S9

Stability of sensor in the determination of blood serum samples

Figure S10

Comparison of the performance of various GOD-based glucose sensors

Table S1

Determination of glucose in blood serum sample

Table S2 


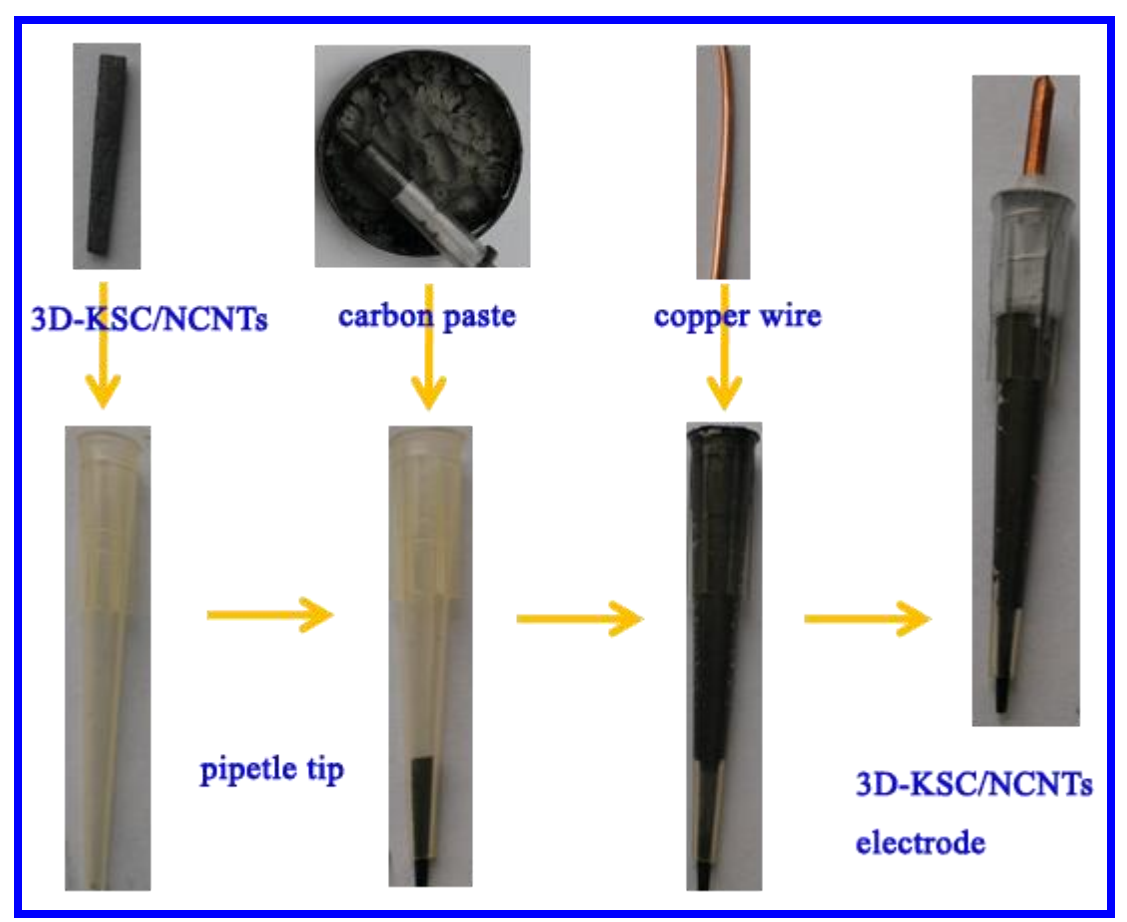

Figure S1. Schematic illustration of the fabrication of the integrated 3D-KSC/NCNTs composite electrode.

As can been seen in Figure S1, a piece of 3D-KSC with appropriate size whose outside diameter is almost equal to the inside diameter of the treated pipette tip (accordingly the 3D-KSC/NCNTs could be immobilized in the treated pipette tip firmly) was inserted into the treated pipette tip leaving only about $0.2 \mathrm{~mm}$ part outside the pipette tip. Then $1.0 \mathrm{~g}$ graphite powder and $0.25 \mathrm{~g}$ liquid paraffin was carefully mixed and homogenized in an agate mortar for approximately $20 \mathrm{~min}$. After that, the homogenized mixture was packed firmly into the above pipette tip to further improve the 3D-KSC/NCNTs immobilization in the pipette. And a copper wire was inserted into the pipette tip to electrically connect with $3 \mathrm{D}-\mathrm{KSC}$ via the graphite powder. Finally, the paste was naturally dried at room temperature before use. 


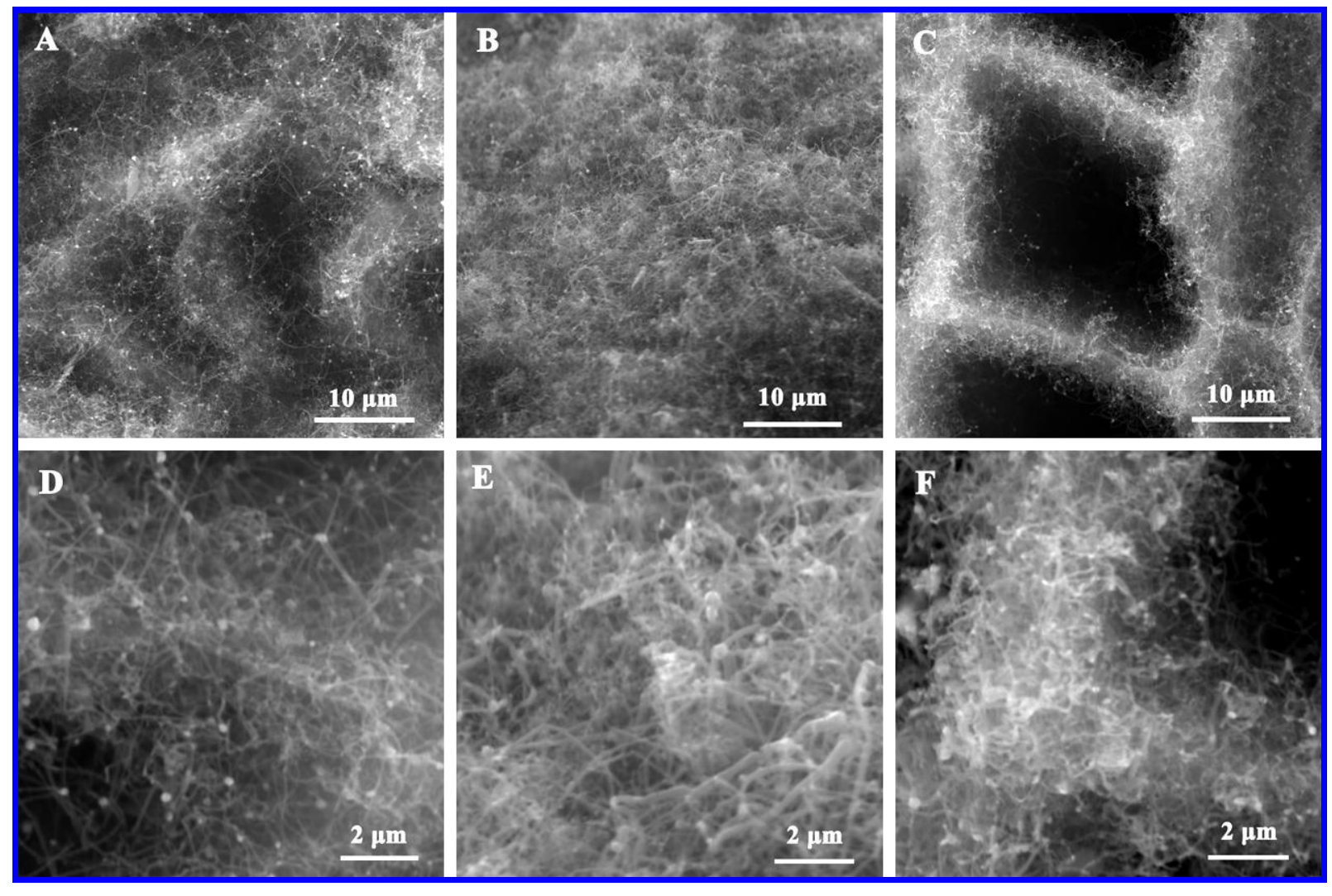

Figure S2. SEM images of the 3D-KSC/NCNTs: (A) top view, (B) side view and (C) cross-section view. (D-F) High magnification images of (A), (B) and (C), respectively. 


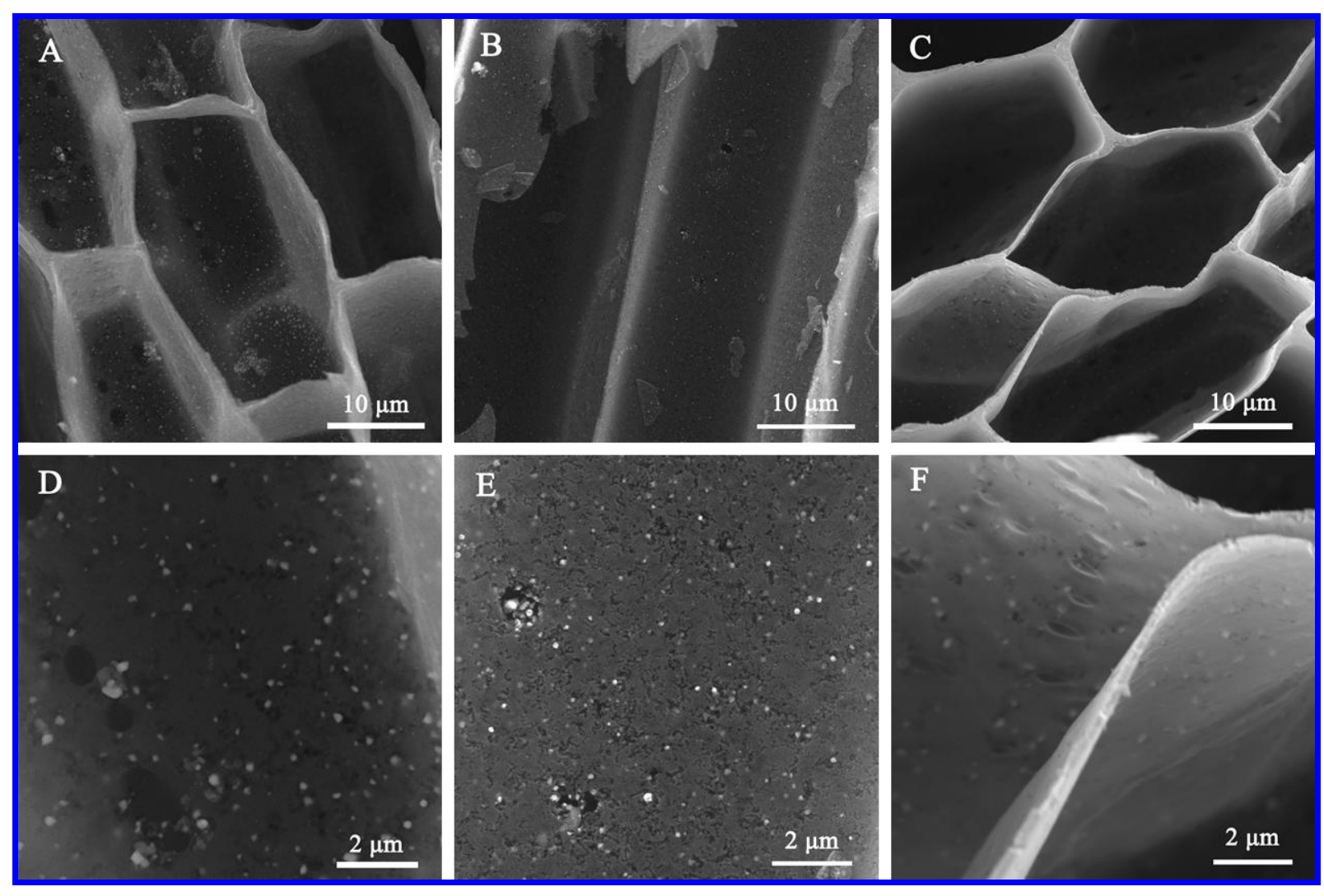

Figure S3. SEM images of the 3D-KSC/Ni NPs: (A) top view, (B) side view and (C) cross-section view. (D-F) High-magnification images of (A), (B) and (C), respectively. 


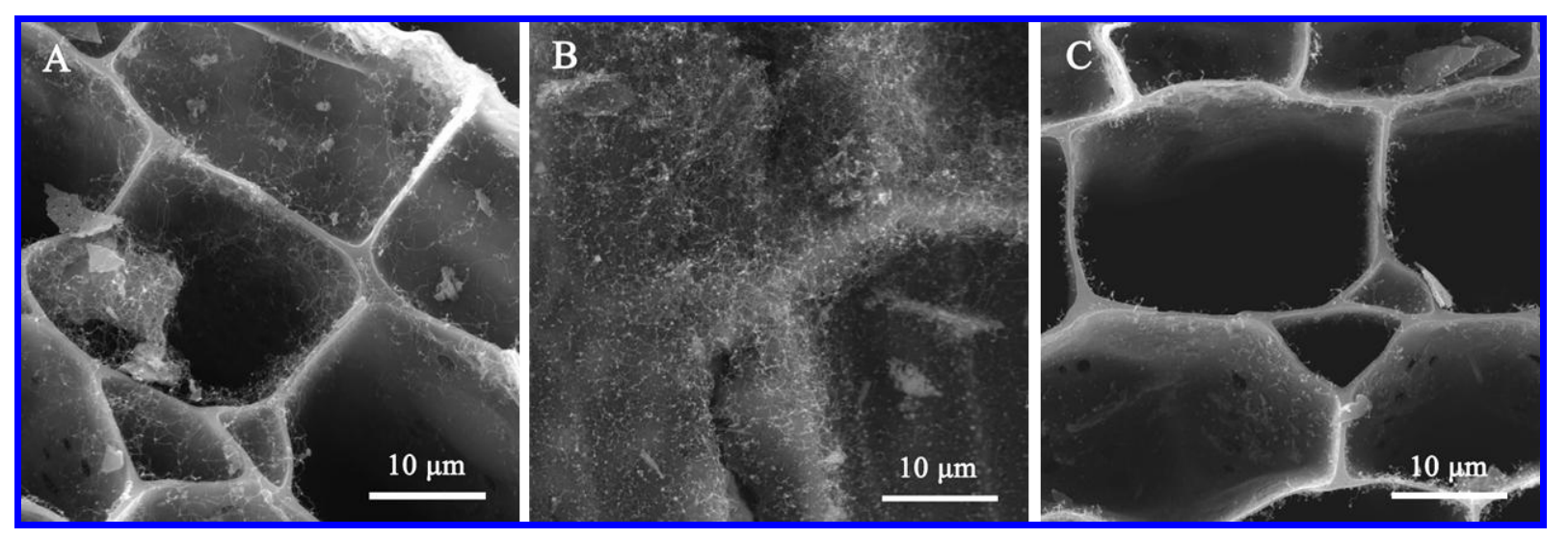

Figure S4. SEM images of the 3D-KSC/NCNTs obtained at a growth time of 20 min: (A) top view,

(B) side view and (C) cross-section view. 


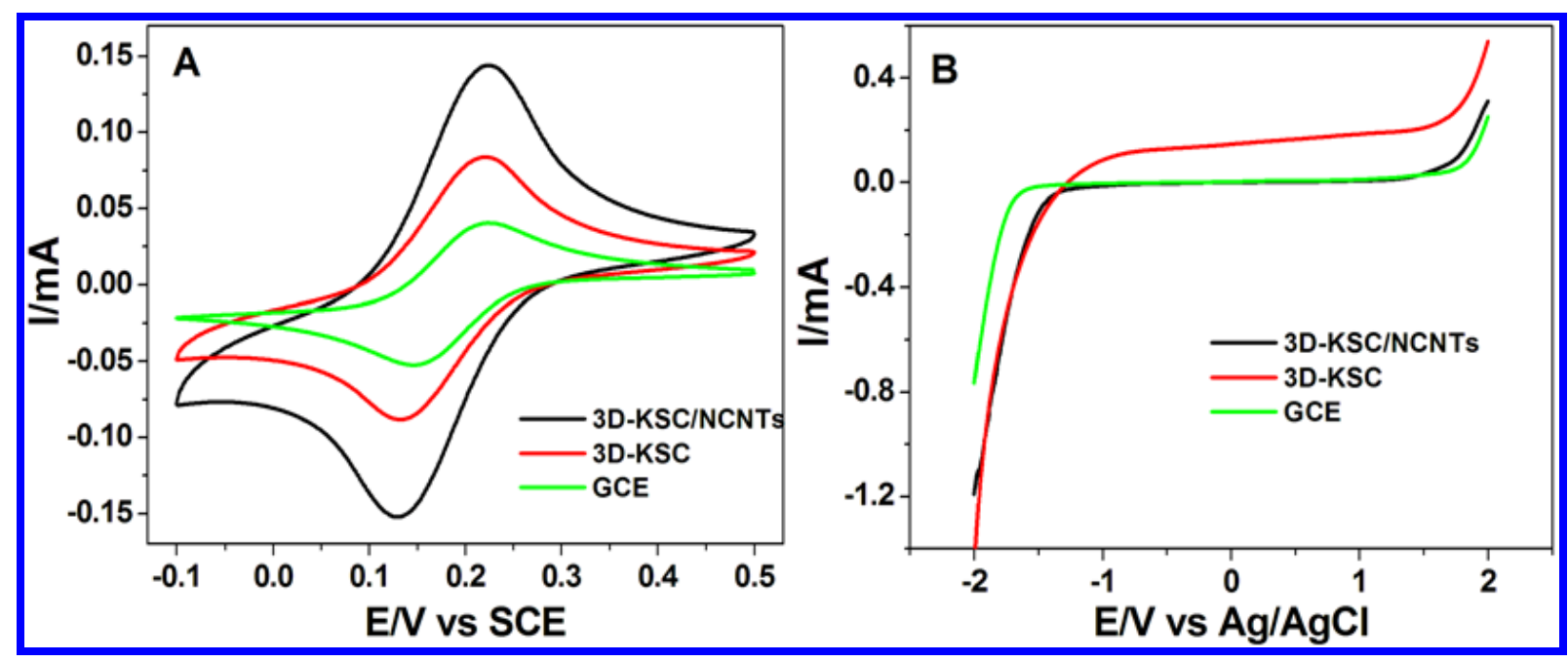

Figure S5. (A) CVs of different electrodes in $0.1 \mathrm{M} \mathrm{KCl}$ solution containing $5.0 \mathrm{mM} \mathrm{K}_{3} \mathrm{Fe}(\mathrm{CN})_{6}$ at scan rate of $50 \mathrm{mV} \mathrm{s}^{-1}$ : bare GCE (green line), 3D-KSC (red line), and 3D-KSC/NCNTs (black line). (B) LSVs of bare GCE (green line), 3D-KSC (red line), and 3D-KSC/NCNTs (black line) in $0.1 \mathrm{M}$ PBS (pH 7.0) at $50 \mathrm{mV} \mathrm{s}^{-1}$. 


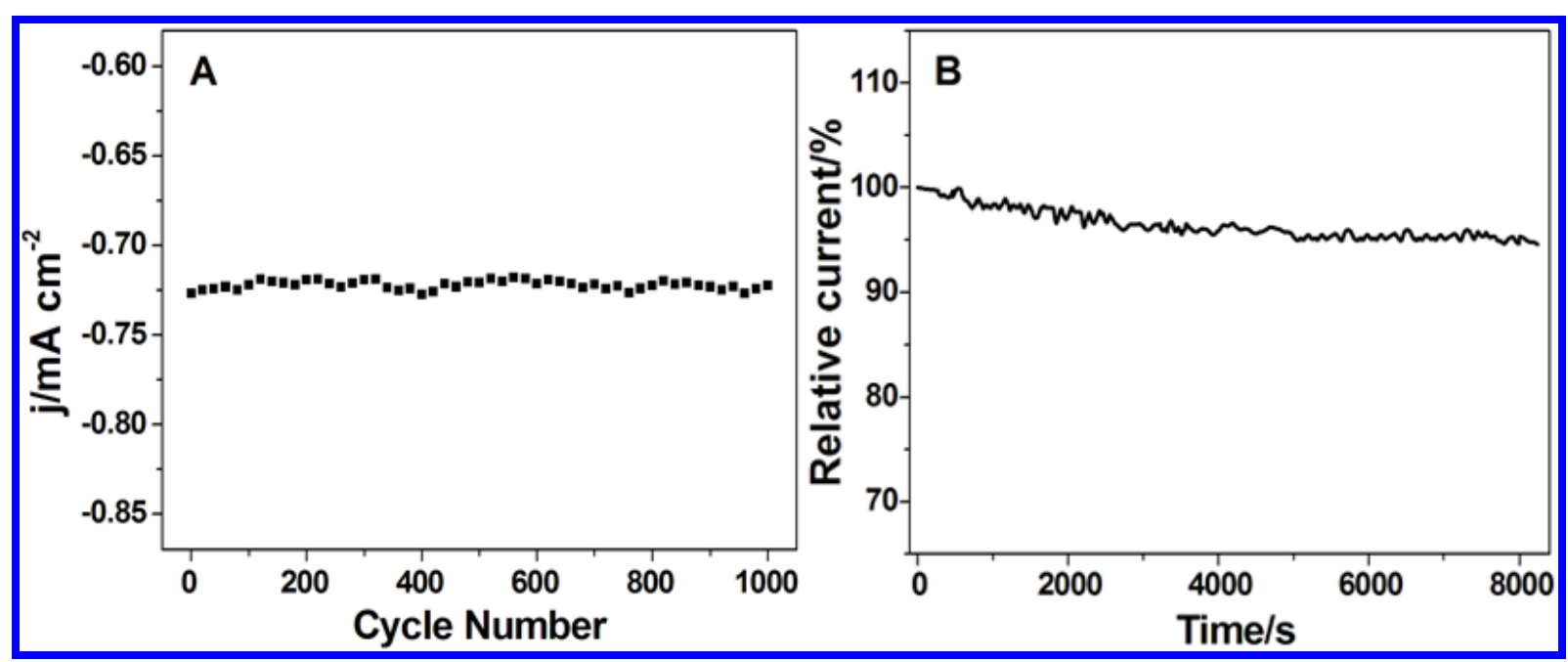

Figure S6. (A) The maximum ORR current density of the 3D-KSC/NCNTs recorded in $\mathrm{O}_{2}$-saturated $0.1 \mathrm{M} \mathrm{KOH}$ solution during repeated cycling. (B) Current-time chronoamperometric response of 3D-KSC/NCNTs electrode at $-0.40 \mathrm{~V}$ in $\mathrm{O}_{2}$-saturated $0.1 \mathrm{M} \mathrm{KOH}$ solution. 


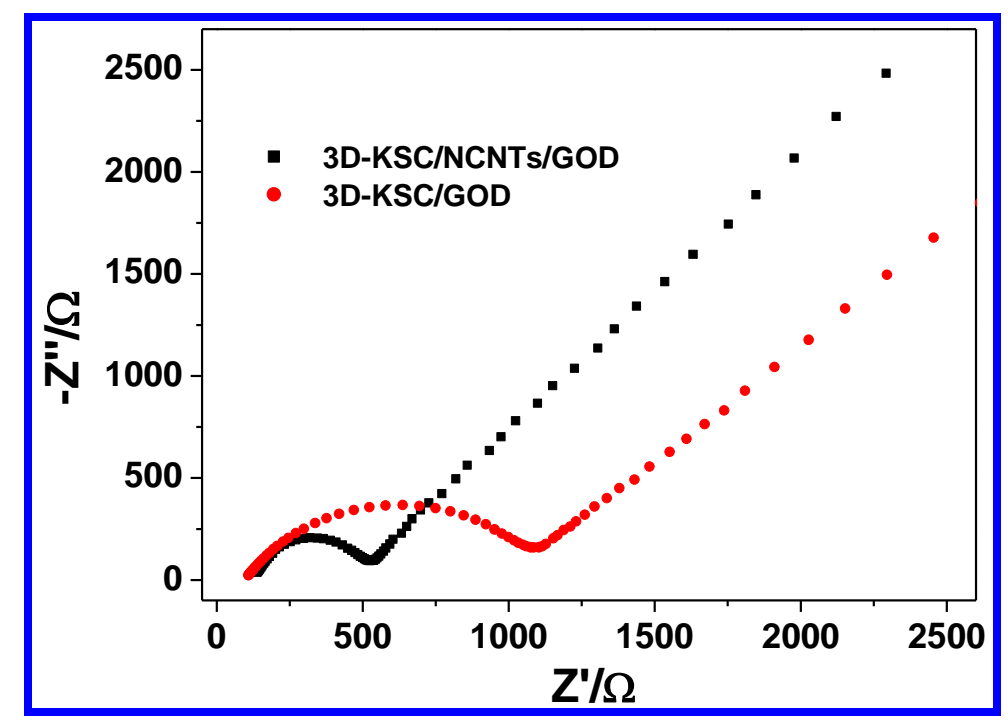

Figure S7. EIS for 3D-KSC/NCNTs/GOD (black line) and 3D-KSC/GOD (red line) in a $0.1 \mathrm{M} \mathrm{KCl}$ solution containing $5.0 \mathrm{mM} \mathrm{Fe}(\mathrm{CN})_{6}{ }^{3-/ 4-}$. 


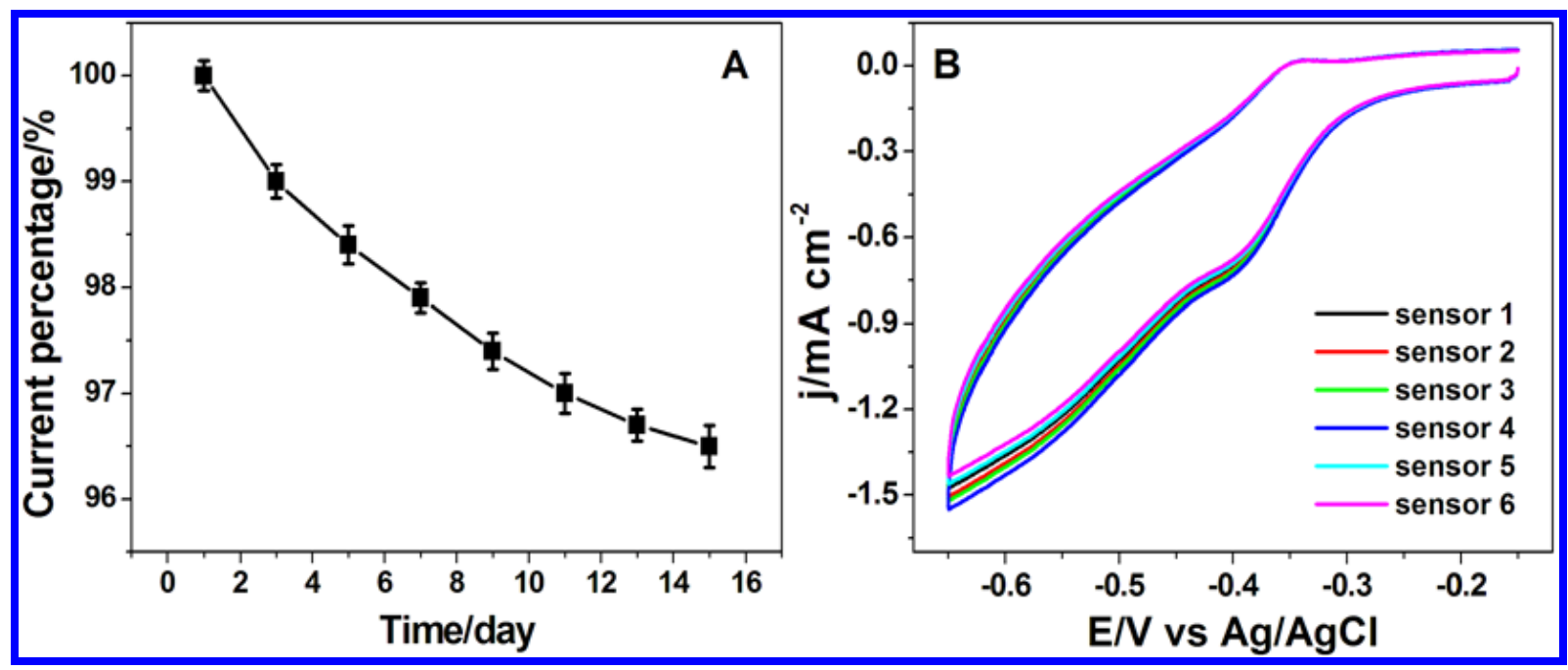

Figure S8. (A) Stability test of the 3D-KSC/NCNTs/GOD electrode in determination of $2.0 \mathrm{mM}$ glucose in 15 days. (B) The responses of six different 3D-KSC/NCNTs/GOD sensors obtained under the same condition toward $2.0 \mathrm{mM}$ glucose. 


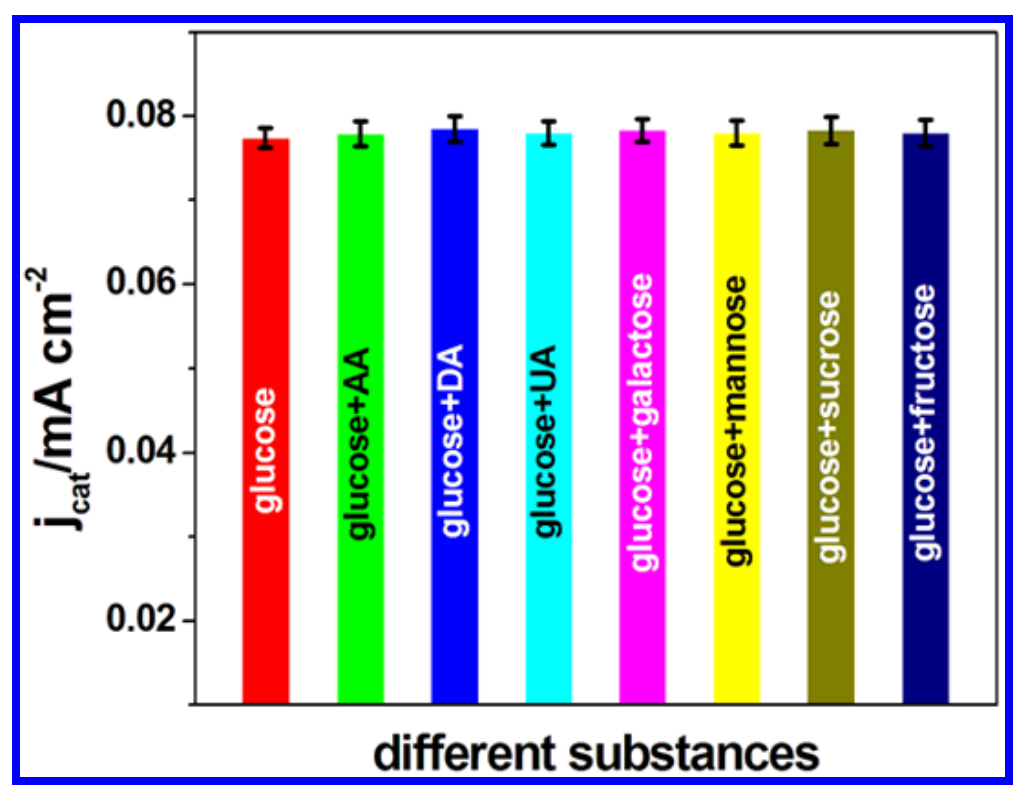

Figure S9. Comparison of the $j_{\text {cat }}$ of the 3D-KSC/NCNTs/GOD with $2.0 \mathrm{mM}$ glucose and 20.0 $\mathrm{mM}$ other interfering substances. 


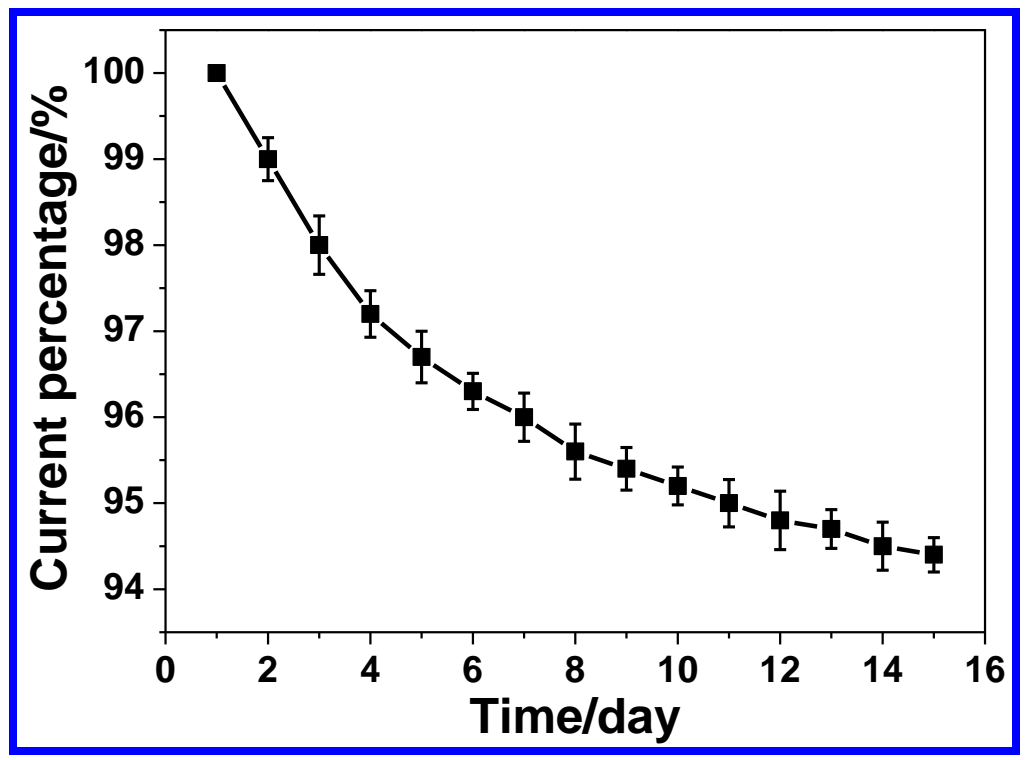

Figure S10. Stability test of the 3D-KSC/NCNTs/GOD in the determination of glucose in blood serum sample. 
Table S1. A comparison of the performance of various GOD-based glucose sensors.

\begin{tabular}{|c|c|c|c|c|}
\hline Glucose sensors & $\begin{array}{l}\text { Detection limit } \\
\mu M\end{array}$ & $\begin{array}{l}\text { Linear range } \\
m M\end{array}$ & $\begin{array}{l}\text { Sensitivity } \\
\mu A \mathrm{~cm}^{-2} \mathrm{mM}^{-1}\end{array}$ & Ref. \\
\hline GOD/graphene-chitosan & 20.0 & $0.08-12.0$ & 37.93 & 1 \\
\hline GOD/Ag-Pdop ${ }^{\mathrm{a}} @ \mathrm{CNTs}$ & 17.0 & $0.05-1.1$ & $3.1 \mu \mathrm{A} \mathrm{mM} M^{-1}$ & 2 \\
\hline $\mathrm{GOD} @ \mathrm{TiO}_{2} / \mathrm{FePc}^{\mathrm{b}}-\mathrm{CNTs}$ & 30.0 & $0.05-4.0$ & $65.3 \mu \mathrm{A} \mathrm{mM}^{-1}$ & 3 \\
\hline GOD/CNTs & 20.0 & $0.04-1.0$ & $2.4 \mu \mathrm{AmM}^{-1}$ & 4 \\
\hline GOD/ERGO ${ }^{\mathrm{c}}-\mathrm{MWCNTs}^{\mathrm{d}}$ & 4.7 & $0.01-6.5$ & 7.95 & 5 \\
\hline GOD/PVA ${ }^{\mathrm{e}}-\mathrm{Au}-\mathrm{pphTEOS}{ }^{\mathrm{f}}$ & 0.7 & $1.0-8.0$ & 43.22 & 6 \\
\hline GOD/Mn-doped ZnS QDs ${ }^{\mathrm{g}}$ & 3.0 & $0.01-0.1$ and $0.1-1.0$ & - & 7 \\
\hline GOD/NCNTs/KSC & 1.9 & $0.0058-18.0$ & 29.4 & This work \\
\hline GOD/KSC & 3.4 & $0.0112-12.0$ & 15.8 & This work \\
\hline
\end{tabular}

a: polydopamine; b: iron phthalocyanine; c: electrochemically reduced graphene oxide; d: multiwalled carbon nanotubes; e: polyvinyl alcohol; f: partially prehydrolyzed tetraethyl orthosilicate; g: quantum dots

The detection limit, $L_{\mathrm{D}}$, is the lowest amount of analyte in a sample that can be detected with a specified degree of certainty (M.A. Castillo, R.C. Castells, Journal of Chromatography A, 921 (2001) 121-133). $L_{\mathrm{D}}$ depends on blank signal $\left(\mu_{\mathrm{b} 1}\right)$ and response dispersion at the blank level $\left(\sigma_{\mathrm{b} 1}\right)$. The detection limit could be estimated according to the following equation: ${ }^{8}$

$L_{\mathrm{D}}=3.29\left(\sigma_{\mathrm{b} 1} / b_{1}\right)$

where $b_{1}$ is the slope of the calibration line.The linear range is the interval of analyte amount over which the method behaves linearly. The quantitation limit $\left(L_{\mathrm{Q}}\right)$ defines the lower end of the linear range; the upper end is usually imposed by instrumental factors. 
In this work, the $\sigma_{\mathrm{b} 1}$ was obtained for 20 successive measurements of $\mu_{\mathrm{b} 1}$. The value of $\sigma_{\mathrm{b} 1}$ is 0.0175. The calibration line is $\mathrm{y}=0.0489+0.0294 \mathrm{x}$. The slope of the calibration line is 0.0294 . In other words, the value of $b_{1}$ is 0.0294 . So, the detection limit is $1.9 \mu \mathrm{M}$. 
Table S2. Determination of glucose in blood serum sample.

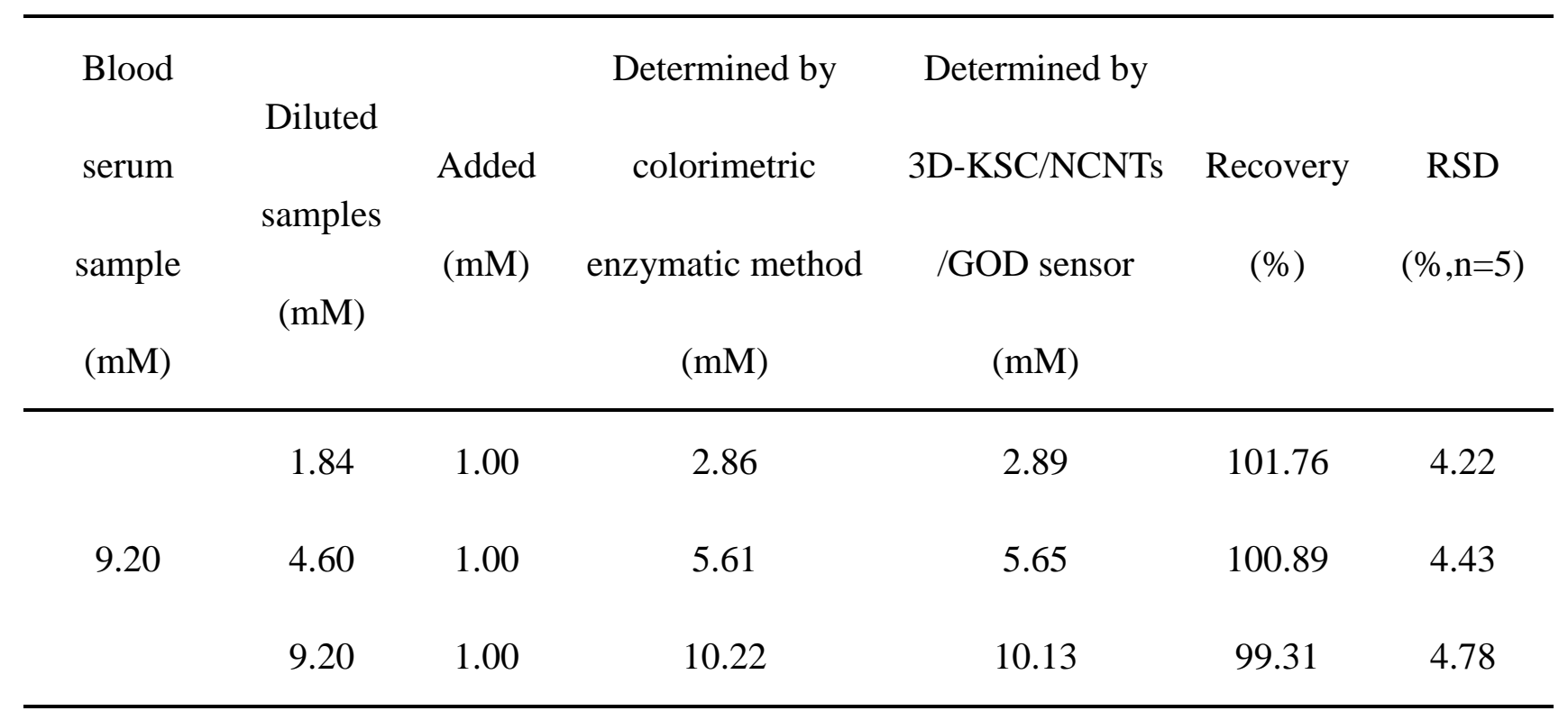




\section{REFERENCE}

(1) Kang, X.; Wang, J.; Wu, H.; Aksay, I. A.; Liu, J.; Lin, Y. Biosens. Bioelectron. 2009, 25, 901-905.

(2) Wang, Y.; Liu, L.; Li, M.; Xu, S.; Gao, F. Biosens. Bioelectron. 2011, 30, 107-111.

(3) Cui, H. F.; Zhang, K.; Zhang, Y. F.; Sun, Y. L.; Wang, J.; Zhang, W. D.; Luong, J. H. Biosens. Bioelectron. 2013, 46, 113-118.

(4) Luo, X.; Killard, A. J.; Smyth, M. R. Electroanalysis 2006, 18, 1131-1134.

(5) Mani, V.; Devadas, B.; Chen, S. M. Biosens. Bioelectron. 2013, 41, 309-315.

(6) Lad, U.; Kale, G. M.; Bryaskova, R. Anal. Chem. 2013, 85, 6349-6355.

(7) Wu, P.; He, Y.; Wang, H. F.; Yan, X. P. Anal. Chem. 2010, 82, 1427-1433.

(8) Castillo,M.A.; Castells, R.C. J. Chromatogr. A 2001, 921, 121-133. 